\title{
Evaluation of Ultra-Microscopic Changes and Proliferation of Apoptotic Glioblastoma Multiforme Cells Induced by Velogenic Strain of Newcastle Disease Virus AF2240
}

\author{
Rola Ali-Saeed ${ }^{1,2 *}$, Aied MAlabsi ${ }^{1}$,Aini Ideris ${ }^{3}$, Abdul Rahman Omar $^{3,4}$, Khatijah \\ Yusoff ${ }^{2}$, Abdul Manaf Ali ${ }^{5,6,7 *}$
}

\begin{abstract}
Aim: Newcastle disease virus (NDV) is a member of genus Avulavirus within the family Paramyxoviridae. Interest of using NDV as an anticancer agent has arisen from its ability to kill tumor cells with limited toxicity to normal cells. Methods: In this investigation, the proliferation of brain tumor cell line, glioblastoma multiform (DBTRG.05MG) induced by NDV strain AF2240 was evaluated in-vitro, by using MTT proliferation assay. Furthermore, Cytological observations were studied using fluorescence microscopy and transmission electron microscopy, DNA laddering in agarose gel electrophoresis assay used to detect the mode of cell death and analysis of the cellular DNA content by flowcytometery. Results: MTT proliferation assay, Cytological observations using fluorescence microscopy and transmission electron microscopy show the anti-proliferation effect and apoptogenic features of NDV on DBTRG.05MG. Furthermore, analysis of the cellular DNA content showed that there was a loss of treated cells in all cell cycle phases (G1, S and G2/M) accompanied with increasing in sub-G1 region (apoptosis peak). Conclusion: It could be concluded that NDV strain AF2240 is a potent antitumor agent that induce apoptosis and its cytotoxicity increasing while increasing of time and virus titer.
\end{abstract}

Keywords: NDV strain AF2240- apoptosis- flow-cytometry- glioblastoma multiforme

Asian Pac J Cancer Prev, 20 (3), 757-765

\section{Introduction}

Brain tumor is formed by abnormal and uncontrolled cell division; it is an abnormal growth of tissue found inside the skull. It can be dangerous because of the importance of the brain and the limited amount of space inside the skull. Most primary brain tumors do not metastasize systemically but spread locally through extensions of infiltrating tumor cells in normal brain. Surgery; radiation and chemotherapy are the current treatments for brain tumor (Henso, 2005; Maher et al., 2001; Stupp et al., 2005; Adam, 2017).

The treatment of primary brain tumors is difficult because of polyclonicity, the blood brain barrier, the diffuse infiltrative nature of these tumors, and the perilous location of some tumors. So, to cure brain tumors some consideration must be taken to kill all cells within the tumor and spare the remaining normal brain cells. Newcastle disease (ND) is a disease of poultry that affecting the alimentary and respiratory tract as well as the central nervous system, it is caused by Newcastle disease virus (NDV) (Brown and Bevins, 2017). Exposure to humans, however, results in mild conjunctivitis, laryngitis and influenza-like symptoms (Othman et al., 2010). Viral therapy for cancer (virotherapy) has significantly been identified to show some promise in cancer therapy. Virotherapy involves the treatment of cancer by using a virus specifically to infect cancer cells while leaving normal cells unharmed (You et al., 2004). NDV is a promise in clinical trials as an antineoplastic agent. It is one of the nonengineered oncolytic viruses, which has a long history as a broad-spectrum oncolytic agent that can destroy tumor cells and stimulate the immune system (Freeman et al., 2006; Schirrmacher et al., 1998). Many preclinical studies indicate that NDV replicates in human cancer cells, but not in normal cells, some types of NDV are able to directly kill certain types of cancer cells and NDV-infected cancer cells can cause the immune system to respond in different ways. Interest in the use of NDV as an anticancer agent has arisen from the ability of the virus to selectively kill human tumor cells with limited toxicity to normal cells. Many strains of NDV (73-T, MH68, Italian, Ulester, Rokin, PV701 and HUJ) have

${ }^{1}$ Faculty of Dentistry, MAHSA University, ${ }^{2}$ Faculty of Biotechnology and Biomolecular Sciences, ${ }^{3}$ Faculty of Veterinary Medicine, ${ }^{4}$ Institute of Bioscience, University Putra Malaysia, ${ }^{5}$ Faculty of Agriculture and Biotechnology, ${ }^{6}$ Institute of Agrobiotechnology, University Sultan Zainalb Abidin, ${ }^{7}$ Natural Medicine Research Centre, University Islam Malaysia, Malaysia. *For Correspondence: rola_absi@yhoo.com 
been shown to exhibit an oncolytic activity (Omar et al., 2003; Freeman et al., 2006). In addition, the oncolytic effects of six Malaysian strains of NDV (AF2240, 01/C, Ijuk, S, F, and V4) have also been studied on several tumor cell lines (Omar et al., 2003; Niederhuber, 2006, Rowa et al., 2017). The goal of most cancer therapy is to reduce the number of tumor cells and to prevent their further accumulation (decrease the rate of cell proliferation). Therefore, in this study the effects of NDV strain AF2240 on the proliferation and the morphological changes of glioblastoma multiforme brain tumor cell line, (DBTRG.05MG), was tested in vitro.

\section{Materials and Methods}

\section{Propagation and Purification of NDV Strain AF2240}

NDV strain was propagated in allantoic fluid of 9-11 days-old embryonated chicken eggs at $37{ }^{\circ} \mathrm{C}$ for 48 hours. The allantoic fluid was harvested, and the presence of virus was confirmed by the haemagglutination test (Alexander,1988). NDV strains AF2240 purified as previously described (Yusoff et al., 1996).

\section{Cells and Cell Culture: A human brain tumor cell line was used in this study}

DBTRG .05MG. Cells were grown as a monolayer in $25 \mathrm{~cm}^{2}$ tissue culture flasks (Nunclon ${ }^{\mathrm{TM}}$, Denmark) at $37^{\circ} \mathrm{C}$ in an atmosphere of $5 \% \mathrm{CO}_{2}$ in RPMI- 1640 medium for DBTRG .05MG supplemented with $10 \%$ fetal calf serum and $1 \%$ antibiotics. Three additional supplements were needed, $2 \mathrm{mM}$ Glutamine, $1 \% \mathrm{HT}$, and $1 \mathrm{mM}$ Sodium Pyruvate. Cells were maintained at $37{ }^{\circ} \mathrm{C}$ in $5 \% \mathrm{CO}_{2}$ atmosphere. Further maintenance and subculturing of cells were done according to supplier's protocol.

\section{The MTT Cell Proliferation Assay}

To confirm anti-proliferative effects of NDV on DBTRG .05MG cells, MTT cell proliferation assay was carried out. In this assay, two different value of virus titer were treated the cells and were prepared together with control. The values chosen were $\mathrm{IC}_{25}$ and $\mathrm{IC}_{50}$ concentrations (132 and $460 \mathrm{HAU} / \mathrm{ml}$ ). Each sample was assayed in triplicate, and control samples include cells without NDV. The cells were treated for 24, 48, and 72 hours. At the end of incubation periods, $20 \mu \mathrm{l}$ of MTT solution ( $5 \mathrm{mg} / \mathrm{ml}$ MTT dissolved in PBS) were added to each well containing cells and the plate was incubated at $37^{\circ} \mathrm{C}$ in an atmosphere of $5 \% \mathrm{CO}_{2}$ for 4 hours. After that, most of the medium was removed, then a volume $100 \mu 1$ of DMSO (dimethyl sulfoxide) was added into the wells to soluble the crystals. Finally, the absorbance was measured by ELISA reader at a wavelength of 570 $\mathrm{nm}$. Graphs (OD of samples against time) were plotted to determine the growth rates of cells in a given values.

\section{Trypan Blue Exclusion Assay}

Trypan Blue Exclusion assay was employed to determine the number of viable cells in cultures. The cells were incubated with different $\mathrm{IC}_{25}$ and $\mathrm{IC}_{50}$ concentrations of NDV AF2240 strain (132 and $460 \mathrm{HAU} / \mathrm{ml}$ ). At $37^{\circ} \mathrm{C}$. The viability of the cells was then determined at the designated time interval. Cells were analyzed by viable cell counts under the Microscope, and the percentage of cell viability was obtained. The results were expressed as the mean percentage of cell viability \pm SEM of triplicate cultures.

\section{Phase Contrast Microscopy}

DBTRG .05MG cells at concentration of $1 \times 10^{5}$ cell $/ \mathrm{ml}$ in $2 \mathrm{ml}$ culture medium containing $10 \%$ FBS was seeded into 6 wells plate (Nunclon TM, Nunc) and was treated with $\mathrm{NDV}$ at $\mathrm{IC}_{50}$. Then, the plates incubated in an atmosphere of $5 \% \mathrm{CO}_{2}$ at $37^{\circ} \mathrm{C}$. The morphological changes were observed after $72 \mathrm{~h}$ by using a phase contrast microscope.

\section{Quantification of Apoptosis Using Propidium Iodide and} Acridine Orange Double staining

DBTRG .05MG cells were stained using propidium iodide (PI) and acridine-orange (AO) double staining according to standard procedures and examine under a fluorescence microscope (Lieca attached with Q-Floro Software) N (Guan et al., 2007). DBTRG .05MG cells were seeded in 6-well plate and incubated at $37^{\circ} \mathrm{C}$ in $5 \% \mathrm{CO}_{2}$ atmosphere. Twenty-four hours later, the medium in each well was removed and replaced with NDV at $\mathrm{IC}_{50}$ concentration dissolved in the culture medium and incubated at $37^{\circ} \mathrm{C}$ in $5 \% \mathrm{CO}_{2}$ atmosphere for 72 hours. After the incubation period, detached cells in the medium were collected and added back to trypsinised adherent cells. The cell suspensions were washed with PBS and then incubated with $5 \mu \mathrm{l}$ of acridine orange $(10 \mu \mathrm{g} / \mathrm{ml})$ and $5 \mu \mathrm{l}$ propidium iodide $(10 \mu \mathrm{g} / \mathrm{ml})$ at a ratio of $1: 1$ in $1 \mathrm{ml}$ of cells and re-centrifuged at 1,000 rpm $/ 5 \mathrm{~min}$. A volume of $10 \mu \mathrm{l}$ of the pellet was pipetted on a slide before putting on the cover slip. Within $30 \mathrm{~min}$, the slide was analyzed using a fluorescent microscope (Leica, Germany). Each experiment was assayed three times $(n=3)$. Viable, apoptotic and necrotic cells were quantified in a population of 200 cells. This assay provides a useful quantitative evaluation and was done three times $(n=3)$.

\section{Ultrastructure Changes on DBTRG.05MG Cells Effected by NDV Strains AF2240 (TEM)}

DBTRG .05MG brain tumor cells were treated with $\mathrm{NDV}$ at $\mathrm{IC}_{50}$ concentration and incubated for 24,48 , and $72 \mathrm{~h}$ at $37^{\circ} \mathrm{C}$. The cultured cells were harvested using trypsin and centrifuged for $10 \mathrm{~min}$ at $1,500 \mathrm{rpm}$. The pellets were fixed in $4 \%(\mathrm{v} / \mathrm{v})$ glutaraldehyde in $0.1 \mathrm{M}$ cacodylate buffer ( $\mathrm{pH} 7.4)$ for $4 \mathrm{~h}$ at $4^{\circ} \mathrm{C}$. The fixed cells were centrifuged, and the pellets were blocked in serum which was later fixed in glutaraldehyde overnight at $4^{\circ} \mathrm{C}$. The specimens were washed in three changes of sodium cacodylate buffer ( $\mathrm{pH}$ 7.4) for 10 min each, postfixed in $1 \%$ osmium tetraoxide at $4^{\circ} \mathrm{C}$. The specimens were then washed in three changes of sodium cacodylate buffer $(\mathrm{pH}$ 7.4) for $10 \mathrm{~min}$ each and dehydrated with a graded series of acetone $(35,50,75,95$, and $100 \%)$. The cells were then infiltrated with acetone and resin and embedded with $100 \%$ resin in beam capsule, and left to polymerize at $60^{\circ} \mathrm{C}$ for $48 \mathrm{~h}$. The area of interest in the embedded cells resin block was chosen to use the toluidine blue staining and 
later examined using a light microscope. The selected area was cut in ultrathin sections using ultra microtome. The sections were placed into a grid and stained with uranyl acetate for $10 \mathrm{~min}$ followed by $50 \%$ filtered acetone, and finally stained using lead which was then washed twice with distilled water. The stained samples were then viewed under transmission electron microscopy (Phillips, Eindhoven, Netherlands).

\section{DNA Fragmentation Assay}

Cells at a concentration of $5 \times 10^{6}$ cells $/ \mathrm{ml}$ were seeded into six-well plate (NunclonTM, Denmark) in $2 \mathrm{ml}$ culture medium with a concentration of $\mathrm{IC}_{50}$ value of virus. Some wells were left without virus to be used as a control. After the $72 \mathrm{~h}$ of incubation, detached cells in the medium were collected and added back to trypsinised adherent cells. The cells were spun down at 1,000 (rpm) for $10 \mathrm{~min}$. The supernatant was discarded, and the pellet was washed with PBS twice. The DNA extraction from treated and untreated cells was carried out according to protocol of a kit for Blood and Cultured Cells from QIAGEN.

Flow cytometry Cell cycle analysis for DBTRG .05MG cells treated with NDV

Cells at a concentration of $5 \times 10^{6}$ cells $/ \mathrm{ml}$ of DBTRG $.05 \mathrm{MG}$ cell line was seeded into six-well plate in $2 \mathrm{ml}$ culture medium with a concentration of $\mathrm{IC}_{50}$ value of virus and were incubated at $37^{\circ} \mathrm{C}$ in an atmosphere of $5 \% \mathrm{CO}_{2}$ for $2 \mathrm{~h}$. Some wells were left with no virus to be used as a control. After the incubation period, the cultured cells were harvested using trypsin and centrifuged for $10 \mathrm{~min}$ at $1,000(\mathrm{rpm})$ at room temperature. Cell pellets were fixed by adding $500 \mu \mathrm{l}$ of $80 \%$ cold ethanol and kept for at least $2 \mathrm{~h}$ at $-20^{\circ} \mathrm{C}$. Cells were pelleted at $1,000(\mathrm{rpm})$ for 10 min, and the ethanol was discarded. The cell pellet was washed with $1 \mathrm{ml}$ (PBS/sodium azide) twice. The pellet was resuspended with $1 \mathrm{ml}$ of $(\mathrm{PBS}+0.1 \%$ triton $\mathrm{X}-100$ $+10 \mathrm{~mm}$ EDTA $+50 \mu \mathrm{g} / \mathrm{ml}$ RNase $+2 \mathrm{ug} / \mathrm{ml}$ Propidium iodide) followed by incubated for to $1 \mathrm{~h}$ at $4^{\circ} \mathrm{C}$. Finally, samples were placed in $12 \times 75$ Falcon tubes and the cell cycle was analyzed by flow cytometer (Beckman Coulter, USA). Each experiment was assayed three times $(n=3)$.

\section{Statistical Analysis}

Data was expressed as mean \pm SEM. Statistical analysis was performed with Student's t-test for data from MTT Proliferation assay, AO/PI staining assay, and flow cytometry. Differences were considered significant at $\mathrm{P}<0.05$.

\section{Results}

The effect of NDV on cells proliferation was studied in vitro, by using the MTT proliferation assay with DBTRG.05MG cell lines. In the assay, both concentrations of virus, $\mathrm{IC}_{50}$ and $\mathrm{IC}_{25}$, were used. Untreated cells

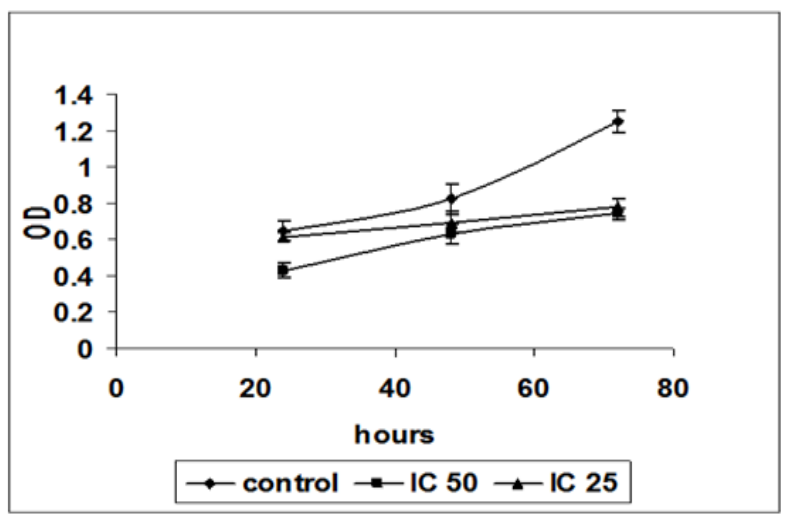

Figure 1. MTT Proliferation Assay for Various Virus Titers $\left(\mathrm{IC}_{50}\right.$ and $\mathrm{IC}_{25}$ ) against DBTRG.05MG Cells at 24, 48 and 72 Hours Post-Inoculation. The growth rates decreased in the treated cells as compared with the untreated cells whereas inoculation with a higher titter of virus $\left(\mathrm{IC}_{50}\right)$ decreased the growth rate more than low titre $\left(\mathrm{IC}_{25}\right)$.
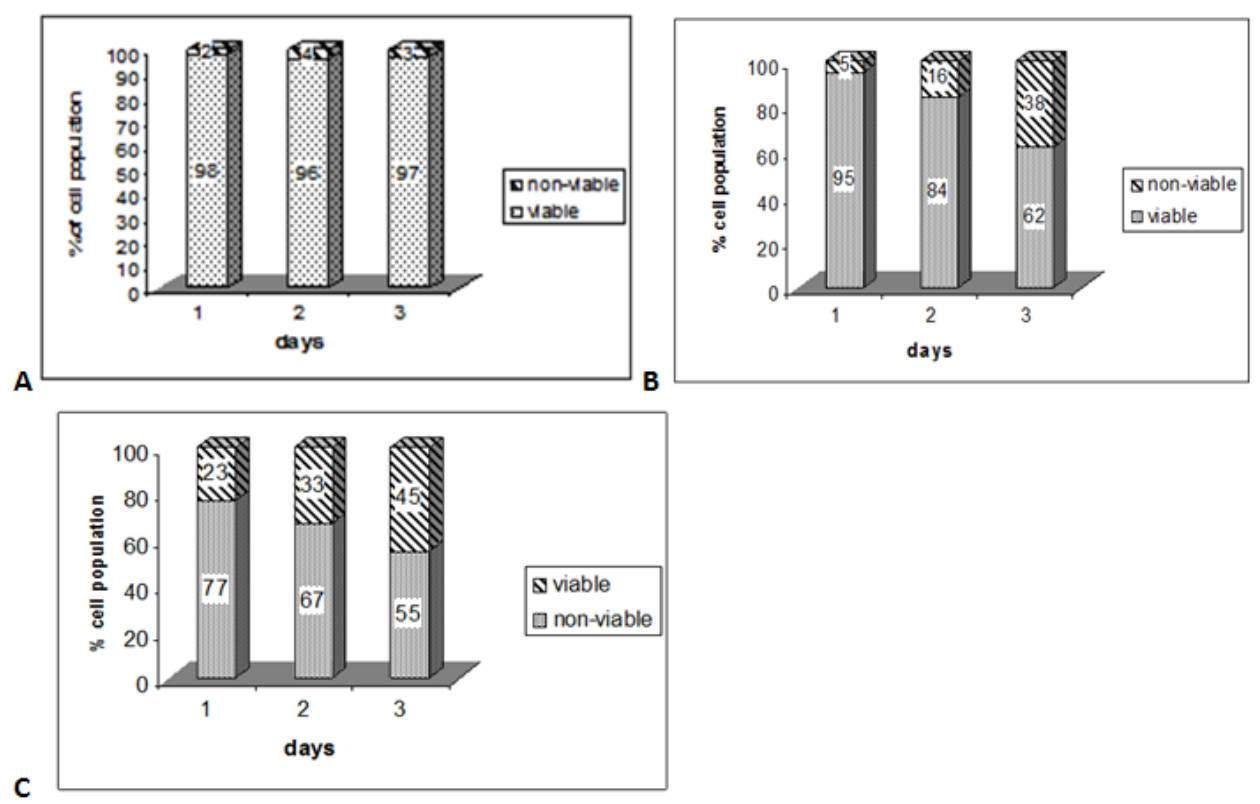

Figure 2. The Percentage of Viable and Non-Viable DBTRG.05MG Cells in Population at Various Time Courses.(A) untreated cells (B) cells treated with $\mathrm{IC}_{25}$ value of NDV AF2240 titer (132 HAU/ml). (C) Cells treated with $\mathrm{IC}_{50}$ value of NDV AF2240 titer (460 HAU/ml). 


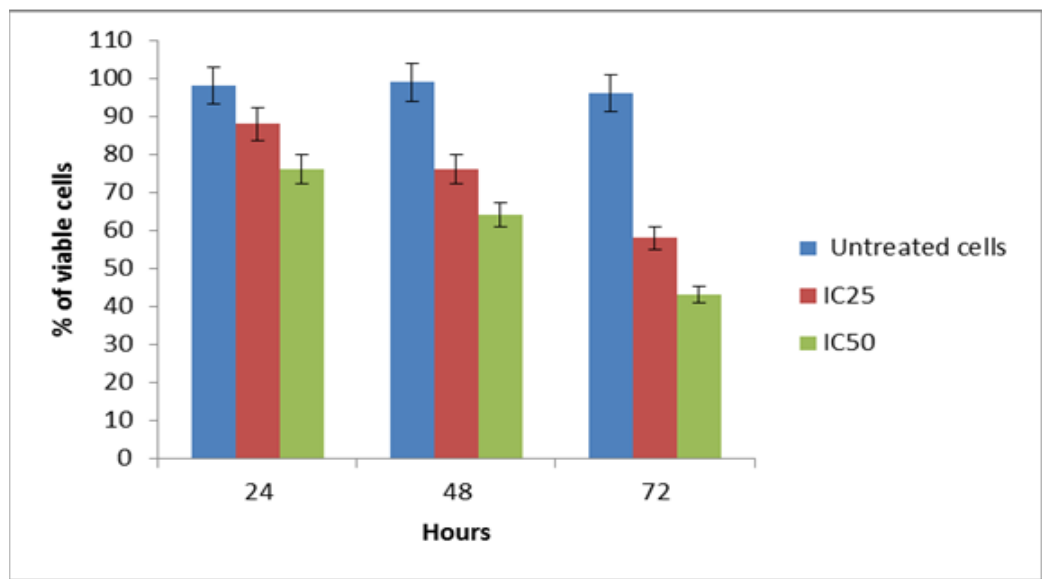

Figure 3. Trypan Blue Exclusion Assay. The percentage of viable cells in DBTRG.05MG cell population after treatment with different concentrations of virus strain at various time intervals.

were used as control. To determine the changes in the numbers of cells in the wells during the experiment, cells proliferation had to be measured 24,48 and 72 hours after the start of the incubation period. Figure 2 showed that the Inoculation of NDV strain AF2240 cells showed lower optical density in both values, $\mathrm{IC}_{25}$ and $\mathrm{IC}_{50}(132$ and $460 \mathrm{HAU} / \mathrm{ml}$ ), than controls. Whereas inoculation of NDV on cells with the $\mathrm{IC}_{50}$ values showed that the optical density was lower than inoculation with the $\mathrm{IC}_{25}$ values. This optical density is in proportion to the number of variable cells. Figures 1 showed that the growth rates

Table 1. Percentage of Apoptotic Cells, Necrotic Cells and Viable DBTRG.05MG Cells under Fluorescent Microscope after 2448 and 72 h Post Inoculation with NDV AF2240. Cells death via apoptosis increased significantly $(\mathrm{P}<0.05)$ in a time-dependent manner.

\begin{tabular}{lccc}
\hline Treatment & $\begin{array}{c}\text { Apoptotic } \\
\text { cells }\end{array}$ & $\begin{array}{c}\text { necrotic } \\
\text { cells }\end{array}$ & $\begin{array}{c}\text { Viable } \\
\text { cells }\end{array}$ \\
\hline Untreated cells after 24 h & 4 & 0 & 96 \\
Untreated cells after 48 h & 5 & 2 & 93 \\
Untreated cells after 72 h & 7 & 3 & 90 \\
treated cells after 24h & 36 & 3 & 61 \\
treated cells after 48h & 54 & 6 & 40 \\
treated cells after 72h & 72 & 7 & 21 \\
\hline
\end{tabular}

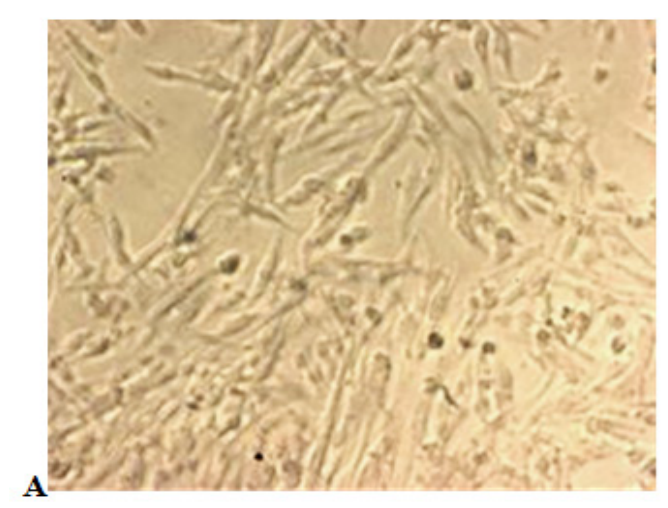

decreased in the treated cells as compared with the untreated cells whereas inoculation with a higher titter of virus $\left(\mathrm{IC}_{50}\right)$ decreased the growth rate more than low titer $\left(\mathrm{IC}_{25}\right)$. On the other hand, the percentage of non-viable DBTRG.05MG cells treated with $\mathrm{IC}_{25}$ value of NDV strain AF2240 was 5\% (day 1), 16\% (day 2) and 38\% (day 3). But the percentage of non-viable DBTRG.05MG cells treated with $\mathrm{IC}_{50}$ values of NDV strain AF2240 were $23 \%$ (day 1), 33\% (day 2) and 45\% (day 3) (Figure 2).

Based on the results of the trypan blue dye exclusion assay, increasing NDV exposure time had a significant effect on DBTRG .05MG cell viability. Treatment of DBTRG .05MG cells with $\mathrm{IC}_{25}$ and $\mathrm{IC}_{50}$ values of virus strain (132 and $460 \mathrm{HAU} / \mathrm{ml}$ ) resulted in a dose- and time-dependent inhibition of cell viability. As observed in Figure 3, at high concentration $\left(\mathrm{IC}_{50}\right)$ of AF2240, DBTRG $.05 \mathrm{MG}$ cell viability reduced to $76 \%$ at $24 \mathrm{~h}$ as compared to control and finally declined to $64 \%$ and $43 \%$ after 48 and $72 \mathrm{~h}$, respectively. At $\mathrm{IC}_{25}$ concentration of AF2240, DBTRG .05MG cell viability reduced to $88 \%$ at $24 \mathrm{~h}$ as compared to control and finally reduced to $76 \%$ and $58 \%$ after 48 and $72 \mathrm{~h}$, respectively.

The morphological changes of DBTRG.05MG cells after treatment with NDV strain AF2240 were observed under Phase Contrast Microscope. The cells were treated with the $\mathrm{IC}_{50}$ value of the virus showed morphological

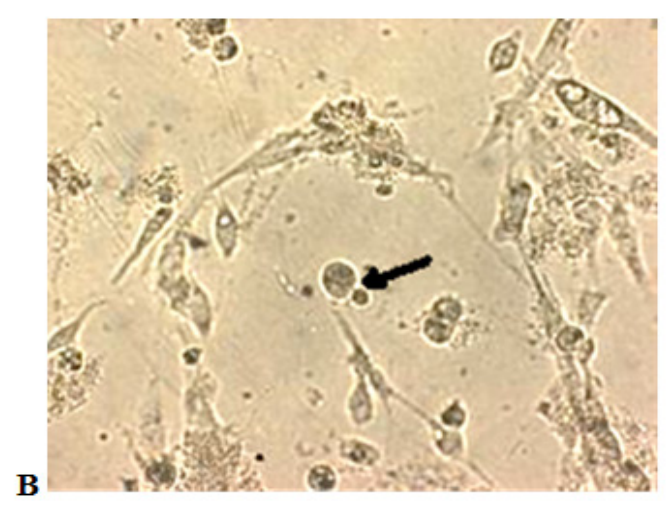

Figure 4. Phase Contrast Microscope Examination of DBTRG.05MG. (Magnification 200X). (A) Untreated DBTRG.05MG cell line, and (B) DBTRG.05MG cell line treated with NDV AF2240 (IC50, 460 HAU/ml) after 72 hours. The virus caused the cells to lose contact with adjacent cells, and cell membrane blebbing (arrow). 

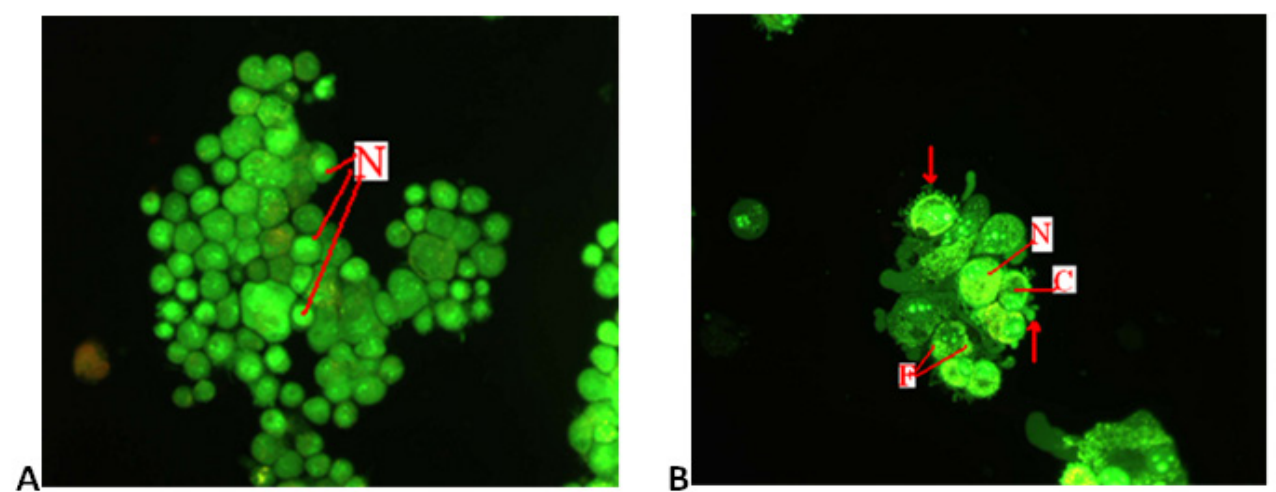

Figure 5. Fluorescence Microscopy Examination of DBTRG.05MG Cell Line. (Magnification 400X). (A) Untreated DBTRG.05MG cells, and (B) DBTRG.05MG cells treated with NDV AF2240 (IC50, 460 HAU/ml) after 72 hours. Viable cells are uniformly green with round nucleus $(\mathrm{N})$. The apoptotic cells are green with condensed chromatin (C) nuclear fragmentation (F) and cell membrane blebbing (arrow).

changes including rounding up of nuclei, shrinkage or decreased of nuclear diameters and condensation of chromatin occurred. Furthermore, the cells in culture lost contact with adjacent cells (Figure 4).

Apoptotic, necrotic, and viable DBTRG.05MG cells were scored under a fluorescence microscope. This is also included the control cells (untreated) whereby, 200 cells were randomly and differentially counted. This study revealed that NDV triggered morphological features that relate to apoptosis in a time-dependent manner (Figure 5 ). Whereby, early apoptosis is obvious by intercalated AO. In several of such cases, the fluorescent bright-green color could be seen in treated cells only. In contrast, untreated cells were observed with a green intact nuclear structure blebbing and nuclear marginations were noticed. Differential scoring of treated DBTRG.05MG cells (200 cells population) showed that there is a statistical significant $(\mathrm{P}<0.05)$ difference in apoptosis positive cells, which indicates clearly that NDV has a time-dependent apoptogenic effect ( Table 1 and Figure 6).

The nucleus, the cytoplasm and the organelles were structurally examined by using TEM for morphological assessment of the nature of cell death. The results showed typical morphological features of apoptotic cells which were occurred 24 hours post-inoculation of the virus on DBTRG.05MG brain tumor cell line. Untreated DBTRG.05MG cells appeared in a normal morphology of viable cells with intact nucleus and clear cytoplasm containing numerous mitochondria and endoplasmic reticulum in their regular shapes. DBTRG.05MG cell lines showed ultrastructural changes after being treated with NDV strain AF2240 for 24, 48 and 72 h. Early stage of apoptosis was observed at 24 hours post-inoculation such as, cell shrinkage, chromatin condensation in dense masses under the nuclear membrane and margination along the inner nuclear membrane, and compaction of the cytoplasm with development of vacuoles in the cytoplasm. At 48 hours post-inoculation, the cells broke up into discrete fragments to form apoptotic bodies and nucleus fragmentation was observed. At 24 hours post-inoculation, cytoplasmic organelles such as intact mitochondria can still be observed whereas at 48 hours post-inoculation numerous mitochondria were found in the centre of the apoptotic cells. Membrane blebbing without disintegration

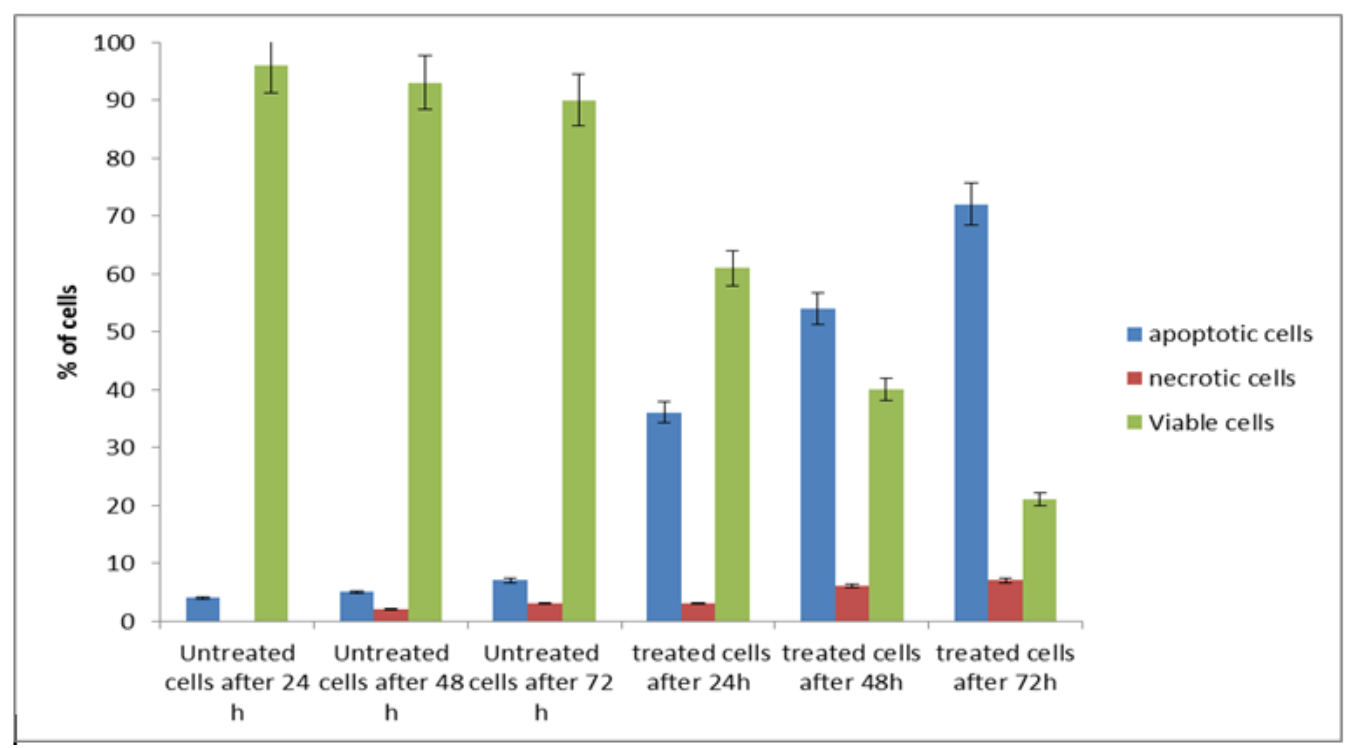

Figure 6. Percentage of Apoptotic Cells, Necrotic Cells and Viable DBTRG.05MG Cells Under Fluorescent Microscope after 2448 and $72 \mathrm{~h}$ Post Inoculation with NDV AF2240. Cells death via apoptosis increased significantly $(\mathrm{P}<0.05)$ in a time-dependent manner. 

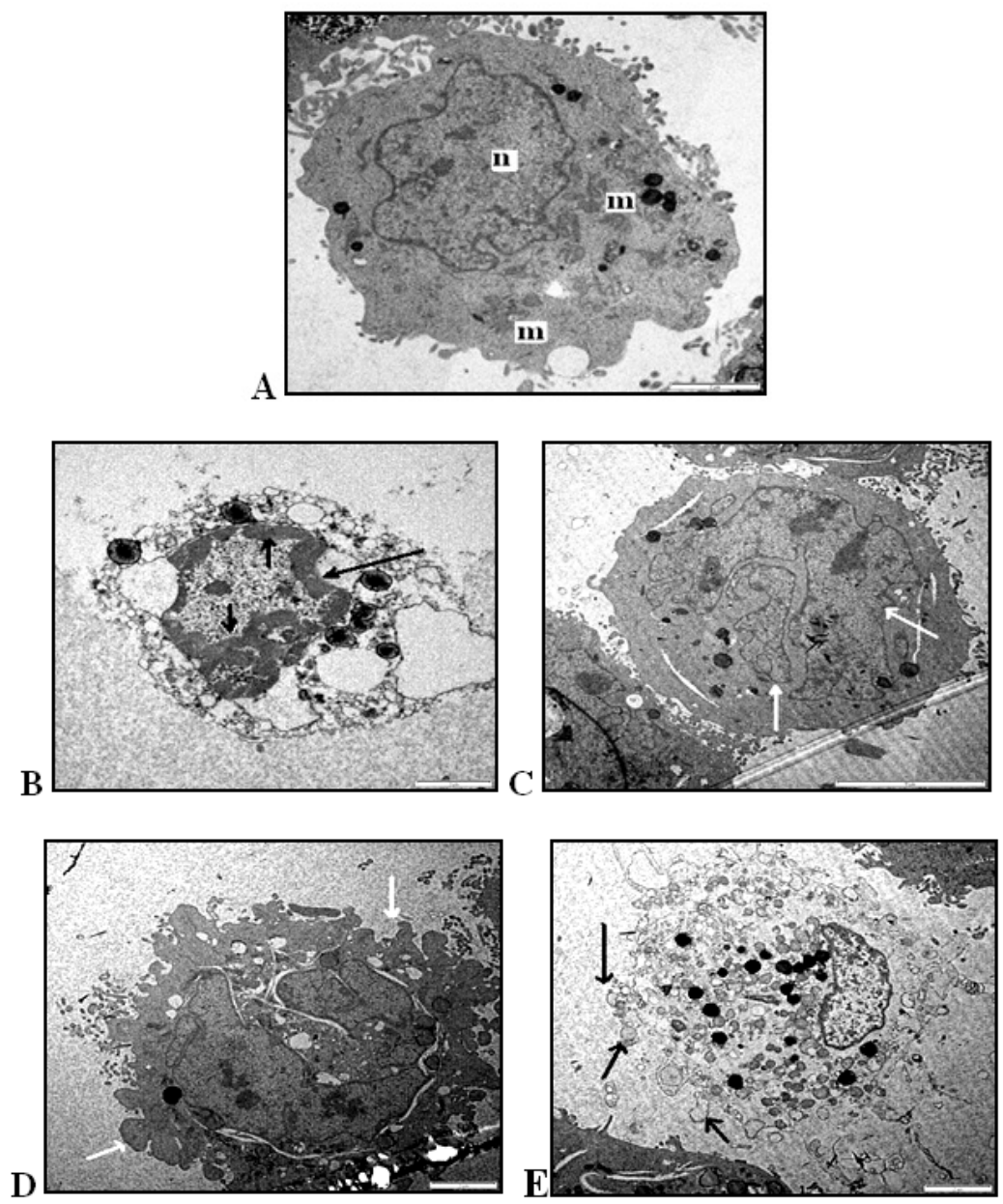

Figure 7. Transmission Electron Micrographs of DBTRG.05MG Cells at Various Stages of Apoptosis (A) untreated DBTRG.5MG cell with intact nucleus (n) mitochondria (m) and clear cytoplasm (Magnification 8000X).(B) chromatin condensed at the nuclear periphery (arrow) (Magnification 8000X). (C) Apoptotic cell containing nuclear fragments (arrow) (Magnification 8000X). (D) Membrane blebbing indicated by arrow (Magnification 12000X).

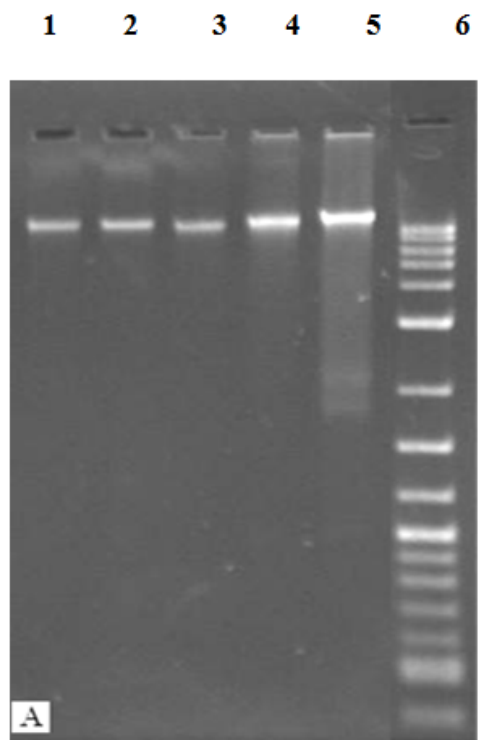

Figure 8. Effects of NDV Strain AF2240 on DNA Fragmentation Extracted from DBTRG.05MG Cell Line at Various Time Periods. Lanes 1, 2 and 3, cells treated with NDV for 72, 48 and 24 hours, respectively; Lane 4, untreated cells; Lane 5, cells treated with doxorubicin for 72 hours (control); Lane 6, $10 \mathrm{~kb}$ marker. of the cellular membrane occurred at 48 and 72 hours post-inoculation indicating of late stage of apoptosis. At 72 hours post -inoculation secondary necrosis was observed, and some apoptotic bodies eventually degenerated (Figure 7).

Fragmentation of chromosomal DNA is the biological hallmark of apoptosis, and can be detected by a ladder formation on gel electrophoresis. The DNA ladder assay is generally accepted as specific for apoptosis because it detects oligonucleosomal cleavage rather than artificial DNA cleavage or necrosis. The DNA of DBTRG.05MG cells treated for 24, 48 and 72 hours with NDV strain AF2240 were extracted and analyzed by agarose gel electrophoresis. Untreated DBTRG.05MG cells contained high-molecular-weight genomic DNA. The inoculation of NDV strain AF2240 on DBTRG.05MG cells at 24, 48 and 72 hours did not show any DNA laddering pattern on the gel (Figure 8).

BTRG.05MG cells were treated with $\mathrm{IC}_{50}$ value of NDV strain AF2240 for 24 and 48 hours, and the DNA content was analyzed by flow cytometry after staining with propidium iodide to determine the effects of NDV on cell cycle distribution. In untreated DBTRG.05MG cells, the $\mathrm{G} 1, \mathrm{~S}$, and $\mathrm{G} 2 / \mathrm{M}$ populations represented $54.34 \%$, $18.66 \%$, and $22.83 \%$ of the cells, respectively (Figure 9). 


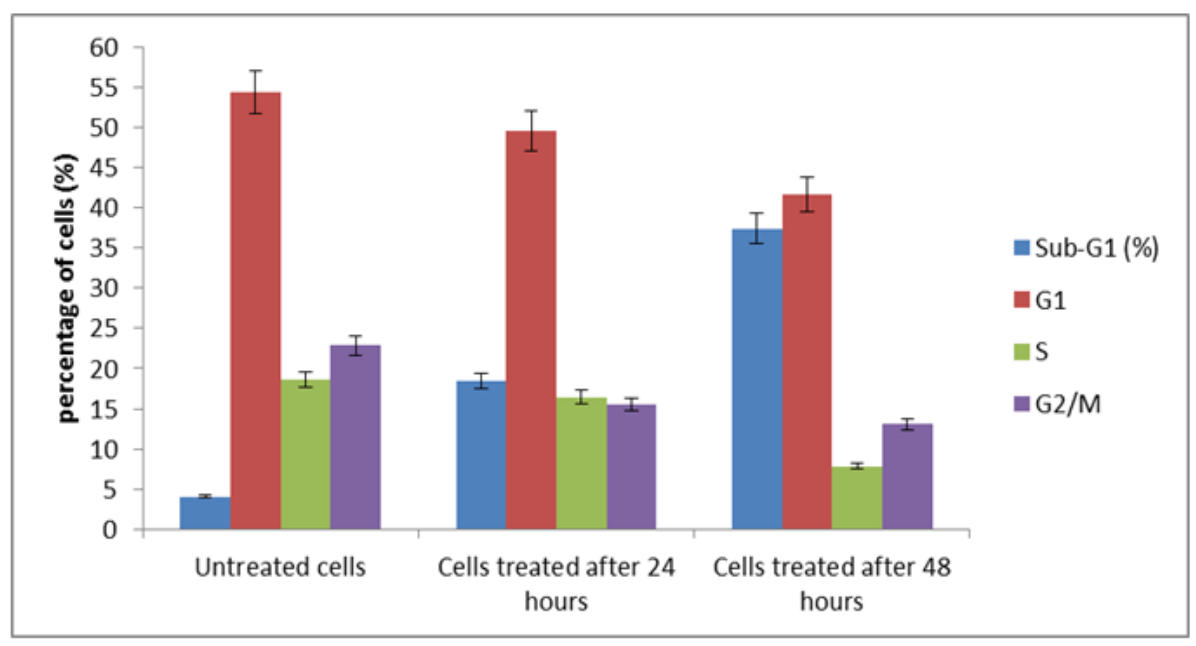

Figure 9. Cell Cycle (DNA Content) Flow Cytometer. Percentage of DBTRG.05MG cells in different cell cycle phase after treatment with IC50 value of NDV strain AF2240. Shows increasing in percentage of sub-G1 (apoptotic) cell population (broken DNA).

The treatment with NDV strain AF2240 did not induce cell cycle arrest in any specific phase. The treatment resulted in a loss of DBTRG.05MG cells in all three phases of the cycle accompanied with a large increase in the sub-G1 region. Apoptosis peak (sub-G1) was found in untreated DBTRG.05MG cells with a small percentage for about $4.09 \%$. The sub-G1 population in DBTRG.05MG cells treated with NDV strain AF2240 was $18.40 \%$ and $37.40 \%$ for 24 and 48 hours post-inoculation, respectively. This virus-induced cell cycle perturbation concurred with the results of the microscopic assays to suggest that brain tumor DBTRG.05MG cell line undergo apoptosis more extensively with increasing in time.

\section{Discussion}

A brain tumor is formed by abnormal and uncontrolled cell division, either found in the brain itself or spread from cancers located in other organs. Grade IV astrocytoma, glioblastoma multiforme (GBM), is the most aggressive form of the primary brain tumors, it is the most common primary brain tumor in adults (Tatter, 2005). The standard treatments include surgery; radiation and chemotherapy are the current treatments for brain tumors (Ali et al., 2011). For three decades, several papers have shown the effectiveness of the NDV and medical literature documented its anticancer activity and its ability to induce tumor lysis through different mechanisms. Cell death can occur by two distinct processes: apoptosis and necrosis (Muneesh et al., 1995). Tumor cells under inappropriate conditions continue to proliferate even in the absence of proliferative signals. The goal of most cancer therapy is to reduce the number of tumor cells and to prevent their further accumulation (decrease the rate of cell proliferation). By using MTT cell proliferation assay this study found that the virus was capable to inhibit proliferation of both cell lines. Furthermore, in this study, cell death caused by NDV strain AF2240 infection on brain tumor cell lines occurred through apoptosis was identified from morphological and ultrastructural observations. Morphological assessment has been the most reliable method for the identification of individual apoptotic cells (Shaghayegh et al., 2018). The morphological features of apoptosis such as cell shrinking, cell membrane blebbing and chromatin condensation were observed by using phase contrast microscopy, fluorescent microscopy (AO/ PI method) and transmission electron microscopy.

Most of the details of the viable cells are undetectable in bright field microscopy, but phase contrast microscopy can be utilized to produce high-contrast images of transparent specimens, such as living cells usually cells in culture. This method was used to observe the cells changing within its media after inoculation of NDV to determine apoptotic features. Phase contrast microscopy showed that treatment of DBTRG.05MG and U-87MG cells with NDV strain AF2240 produces fewer adherent cells compared to controls, and a larger proportion of freely floating cells. The cells rounded up, and the cells in culture lost contact with adjacent cells during treatment, and are therefore more likely to be shed from the plate. This occurs during which time any specialized surface structures such as microvilli disappeared and convolutions began to form (Liu and Liu, 2001). Rounding up of nuclei was one of the morphological changes occurred because of the digesting of the protein structures, which conform the cytoskeleton, by specialized peptidases (known as caspases) that have been activated inside the cell (Liu and Liu, 2001).

Chromatin condensation, nuclear shrinkage and formation of apoptotic bodies can easily be observed under fluorescence microscopy, after appropriate staining of nuclei with DNA-specific fluorochromes (Clarke et al., 2000). In this study, the brain tumor cell line DBTRG.05MG were treated with NDV strain AF2240 then stained with acridine orange and propidium iodide $(\mathrm{AO} / \mathrm{PI}) . \mathrm{AO}$ is a membrane-permeable cationic dye that binds to nucleic acids of viable cells, and at low concentrations it causes green fluorescence. PI is impermeable to intact membranes, but readily penetrates the membranes of nonviable cells and binds to DNA or RNA, causing orange fluorescence. Propidium iodide used for analysis of nuclear morphology and Acridine Orange 
(AO) a metachromatic dye which differentially stains double-stranded (ds) and single-stranded (ss) nucleic acids. When AO intercalates into dsDNA it emits green fluorescence upon excitation at $480-490 \mathrm{~nm}$.

When cells were viewed under fluorescence microscopy, it was observed that untreated cells had round intact nuclei and stained green indicating viable cells whereas the treated cells stained green and exhibited features of apoptotic cells, which had irregular shaped nucleus, shrinkage, condensed chromatin and cell membrane blebbing (Chan et al., 2006).

Transmission electron microscopy Briefly, after treatment of DBTRG.05MG cells with NDV strain AF2240, some cells showed typical morphologic changes of apoptosis, including shrinkage and membrane blebbing. Intracellular and plasma membrane structural modifications have been widely recognized as crucial factors involved in cell injury and death. Changes in nuclear morphology and in organelle structure as well as specific phenomena at the cell surface, namely surface smoothing and surface blebbing, are often considered as markers associated with cell pathology (Malorni 1998). TEM analysis could reveal the changes of cell ultrastructure during the apoptotic process (Lin et al., 2004). By means of TEM the results in this study showed that untreated brain tumor cells showed no changes and exhibited intact nucleus with clear cytoplasm while after brain tumor cell lines treating with NDV strain AF2240 the typical morphological features of apoptotic cells appeared, including chromatin condensation in dense masses under the nuclear membrane, compaction of the cytoplasm, crowding of organelles and surface protuberances. These changes occur within the nucleus during apoptotic death such as the appearance of dense, crescent-shaped chromatin aggregates which line nuclear membrane. Later, the nucleolus disintegrates; nuclear membrane develops deep invaginations and, ultimately, the nucleus fragmented into dense granular particles (apoptotic bodies). It is thought that the nuclear changes are due to activation of endogenous nuclease(s) which cleaves DNA into oligonucleosomal fragments (Oberhammer et al., 1993; Sho et al., 2001).

At present, DNA fragmentation is considered the most characteristic feature of programmed cell death as shown by studies of cells in culture (Gavrieli et al., 1992). DNA ladder assay provides a sensitive assay for the detection of DNA fragmentation, but this method is qualitative rather than quantitative (Muneesh et al., 1995). Fragmentation of chromosomal DNA is the biological feature of apoptosis, and can be detected by a ladder formation pattern using gel electrophoresis. The DNA ladder assay is generally accepted as specific for apoptosis because it detects oligonucleosomal cleavage rather than artificial DNA cleavage or necrosis. To date, apoptosis has been characterized biochemically by the production of 180-200 bp internucleosomal DNA fragments resulting from the activation of an endonuclease (Lin et al., 2004).

In this study, we reported that NDV strain AF2240 did not stimulate DNA fragmentation characteristic of apoptosis in DBTRG.05MG cell line at 24, 48 and 72 hours post-inoculation. The problem with the ladder assay is its low sensitivity. Even with enhancement of the fragmented DNA in the sample, DNA ladder formation is observed only when the extent of oligonucleosomal cleavage is prominent. Internucleosomal cleavage of DNA is likely to be in the later phase of apoptotic process (Lin et al., 2004; Cohen et al., 19921; Gooch and Yee, 1999). In most cell types, the biochemical characteristics of apoptotic response include activation of endogenous calcium and magnesium dependent endonucleases, leading to fragmentation of the chromosomal DNA. Initially, the DNA fragments are large $(50-300 \mathrm{~kb})$ but are later digested to oligonucleosomal size (multimers of 180-200 bp). The formation of this distinct DNA ladder is considered to be a biochemical hallmark of apoptosis. A study by Oberhammer et al., (1993) stated that cleavage of DNA to $50 \mathrm{kbp}$ internucleosomal fragments instead of degradation into 180-200 bp fragments was observed in several epithelial cell types. This was further confirmed by Grem et al., (1996) which stated that DNA fragmentation in some breast cancer cell lines was not companions by the generation of oligonucleosomal laddering; instead, induction of very high molecular weight DNA fragmentation was observed. Therefore, it was reported that apoptosis can occur fully in the absence of DNA fragmentation.

Further confirmation of the mode of cell death was carried by Flowcytometric analysis of cell cycle. It is rapid and quantitatively measures on apoptotic cells. It measures apoptotic changes in cells by staining with DNA dyes (Telford et al., 1994). This method is useful for quantitative estimates of the fractions of cells in the different phases of the cell cycle (Fried et al., 1976). Untreated and treated DBTRG.05MG brain cells were evaluated for apoptosis by measuring the amount of apoptotic cells using of DNA flow cytometry (FCM). NDV strain AF2240 caused an increasing in the subG1 region which increased with increasing of time and did not induce specific cell cycle arrest in a specific phase.

Apoptotic cells, due to a change in membrane permeability, showed an increased up-take of the vital dye compared to live cells. The apoptotic cells with degraded DNA were represented in so-called "sub-G1" peaks on DNA histograms. The percentage of sub-G1 cells was measured. PI is added to discriminate late apoptotic or necrotic cells which had lost membrane integrity from early apoptotic cells which still had intact membranes by dye exclusion (Nicoletti et al., 1991).

Therefore the results show that NDV strain AF2240 was capable to induce apoptosis on DBTRG.05MG brain tumor cell line as demonstrated by the occurrence of various apoptotic features after treatment with the virus.

\section{Acknowledgments}

This research was funded by the National Cancer Council (MAKNA), Malaysia. The authors also acknowledge additional support from Universiti Putra Malaysia, Malaysia. 


\section{References}

Adam R (2017). Types, symptoms, and treatment of a brain tumor. Medical News today. https://www.medicalnewstoday. com/articles/315625.php. 2017, 5 February

Alexander DJ (1988). Newcastle Disease. In Newcastle Disease Virus an Avian Paramyxovirus; KluwerAcademic Publishers: Dordrecht, the Netherlands, pp 1-22.

Brown VR, Bevins SN (2017). A review of virulent Newcastle disease viruses in the United States and the role of wild birds in viral persistence and spread. Vet Res, 2017, 1-15.

Chan KM, Rajab NF, Ishak MHA, et al (2006). Goniothalamin induces apoptosis in vascular smooth muscle cells. Chem Biol Interact, 159, 129-40.

Clarke RG, Lund EK, Johnson IT, Pinder AC (2000). Apoptosis can be detected in attached colonic adenocarcinoma HT29 cells using Annexin V binding, but not by TUNEL assay or sub-G0 DNA content. Cytometry, 39, 141-50.

Cohen GM, Sun XM, Snowden RT, Dinsdale D, Skilleter DN (1992). Key morphological features of apoptosis may occur in the absence of internucleosomal DNA fragmentation. Biochem J, 286, 331-4.

Freeman AI, Zakay-Rones Z, Gomori JM, et al (2006). Phase I/ II trial of intravenous NDV-HUJ oncolytic virus in recurrent glioblastoma multiforme. Mol Ther, 13, 221-8.

Fried J, Perez AG, Clarkson BD (1976). Flow cytofluorometric analysis of cell cycle distributions using propidium iodide, Properties of the method and mathematical analysis of the data. J Cell Biol, 71, 172-81.

Gavrieli Y, Sherman Y, Ben-Sasson SA (1992). Identification of programmed cell death in situ via specific labeling of nuclear DNA fragmentation. J Cell Biol, 119, 493-501.

Gooch JL, Yee D (1999). Strain-specific differences in formation of apoptotic DNA ladders in MCF-7 breast cancer cells. Cancer Lett, 144, 31-7.

Guan T, Qin F, Du J, et al (2007). AICAR inhibits proliferation and induced S-phase arrest, and promotes apoptosis in CaSki cells. Acta Pharmacol Sinica, 28, 1984-90.

Henso JW (2005). Glioblastoma multiforme and anaplastic glioma: a patient guide. http://brain.mgh.harvard.edu/ PatientGuide 2005. htm. Accessed on $7 \mathrm{Feb}$.

Lin W, Li D, Chen Q, Lu H (2004). Clinical and experimental study of oxaliplatin in treating human gastric carcinoma. World J Gastroenterol, 19, 2911-5.

Liu C, Xu HY (2001). Induction of caspase-dependent apoptosis in cultured cells by the avian coronavirus infectious bronchitis virus. Am Soc Microbiol, 75, 6402-9.

Maher EA, Furnari FB, Bachoo RM, et al (2001). Malignant glioma: genetics and biology of a grave matter. Genes Dev, 15, 1311-33.

Malorni W, Fais S, Fiorentini C (1988). Morphological aspects of apoptosis. Purdue Cytometry, CD-ROM 4. 1998.

Muneesh T, Vishva MD (1995). Fas- and tumor necrosis factorinduced apoptosis is inhibited by the poxvirus crmA gene product. Am Soc Biochem Mol Biol, 270, 3255- 60.

Nicoletti I, Migliorati G, Pagliacci MC, Grignani F, Riccardi C (1991). A rapid and simple method for measuring thymocyte apoptosis by propidium iodide staining and flow cytometry. J Immunol Methods, 139, 271-9.

Niederhuber JE (2006). Questions and answers about Newcastle disease virus. http://www.cancer.gov/cancertopics/pdq/cam/ NDV/ Patient/page2. 2006; Accessed 16 March.

Oberhammer F, Wilson JW, Dive C, et al (1993). Apoptotic death in epithelial cells: cleavage of DNA to 300 and/or 50 $\mathrm{kb}$ fragments prior to or in the absence of internucleosomal fragmentation. $E M B O J, \mathbf{1 2}, 3679-84$.

Omar A, Aini I, Ali A, et al (2003). An overview on the development of Newcastle disease virus as an anti-cancer therapy. Malays J Med Sci, 10, 4-12.

Othman F, Ideris A, Motalleb G, Eshak ZB, Rahmat A (2010). Oncolytic effect of Newcastle disease virus AF2240 strain on the MCF-7 breast cancer cell line. Yakhteh Med J, 12, 17-24.

Rowa A, Aied Mohammed A, Abdul Manaf A, Gumballi S (2017). Anti-tumor activity of Newcastle Disease Virus Strains (AF 2240 and V4-UPM) on AOM-induced ACF in sprague-dawley rats. Int J Acad Res Dev, 7, 11461-7.

Schirrmacher V, Ahlert T, Pröbstle T, et al (1998). Immunization with virus-modified tumor cells. Semin Oncol, 25, 677-96.

Shaghayegh G, Alabsi AM, Ali-Saeed R, et al (2017). Effects of damnacanthal and nordamnacanthal on proliferation, apoptosis, and migration of oral squamous cell carcinoma cells. Asian Pac J Cancer Prev, 18, 3333-41.

Sho E, Sho M, Singh TM, et al (2001). Blood flow decrease induces apoptosis of endothelial cells in previously dilated arteries resulting from chronic high blood flow. Arter Thromb Vasc Biol, 21, 1139- 45.

Stupp R, Mason WP, van den Bent MJ, et al (2005). Radiotherapy plus concomitant and adjuvant temozolomide for glioblastoma. N Engl J Med, 352, 987-96.

Tatter SB (2005). The new WHO Classification of Tumors affecting the Central Nervous System. http://neurosurgery. mgh.harvard.edu/newwhobt.htm. 2005; Accessed on 11 May.

Telford WG, King LE, Fraker PJ (1994). Rapid quantitation of apoptosis in pure and heterogeneous cell populations using flow cytometry. J Immunol Methods, 172, 1-16.

You L, He B, Xu Z, McCormick F, Jablons DM (2004). Future directions: oncolytic viruses. Clin Lung Cancer, 5, 226-30.

Yusoff K, Tan WS, Lau CH, Ng BK, Ibrahim AL (1996). Sequence of the haemagglutinin-neuraminadase gene of the Newcastle disease virus oral vaccine strain V4 (UPM). Avian Pathol, 25, 837-44.

Fisher B, Taphoorn MJB, Belanger K, et al (2005). Radiotherapy plus concomitant and adjuvant temozolomide for glioblastoma. N Engl J Med, 352, 987-96.

Tatter SB (2005). The new WHO Classification of Tumors affecting the Central Nervous System. http://neurosurgery. mgh.harvard.edu/newwhobt.htm. 2005; Accessed on 11 May.

Telford WG, King LE, Fraker PJ (1994). Rapid quantitation of apoptosis in pure and heterogeneous cell populations using flow cytometry. J Immunol Methods, 172, 1-16.

You L, He B, Xu Z, McCormick F, Jablons DM (2004). Future directions: oncolytic viruses. Clin Lung Cancer, 5, 226-30.

Yusoff K, Tan WS, Lau CH, Ng BK, Ibrahim AL (1996). Sequence of the haemagglutinin-neuraminadase gene of the Newcastle disease virus oral vaccine strain V4 (UPM). Avian Pathol, 25, 837-44

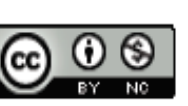

This work is licensed under a Creative Commons AttributionNon Commercial 4.0 International License. 